\title{
A REGIOSELECTIVE SYNTHESIS OF 6-ARYLAZO-7-HYDROXYCOUMARINS AND 5"- ARYLAZOPSORALENS.
}

R.V. Rozhkov, A.V. Vasilyev, V.F. Traven*, D.I.Mendeleev University of Chemical Technology of Russia. Moscow 125190, Russia;

E.A. Carberry, Southwest State University, Marshall. MN 56258.

\begin{abstract}
Azocoupling reactions of 7-hydroxycoumarins and psoralens with para-substituted aryldiazonium tetrafluoroborates have been studied in the presence of sodium hydroxide solution. The reaction is highly regioselective to yield only 6-arylazo-7-hydroxycoumarins and 5'-arylazopsoralens. The key step of the reaction is pyrone ring opening which activates positions 6 and $5^{\prime}$ of the coumarins and psoralens respectively in electrophilic substitution. Reduction of 6-arylazo-7-hydroxy-4-methyl coumarin provides a convenient route to 6 -amino-7-hydroxy-4-methylcoumarin with $70 \%$ overall yield.
\end{abstract}

\section{Introduction}

Many 7-hydroxycoumarin derivatives show anticancer [1,2], anticoagulant [3] and antibacterial [4] activities. Some furocoumarins such as 5-methoxypsoralen, 8-methoxypsoralen and 4.8,5'-trimethylpsoralen are currently used in PUVA therapy [5] to treat a variety of skin diseases, such as psoriasis, mycosis fungoides, etc. Therefore, chemical transformations of 7-hydroxycoumarins and furocoumarins are much of interest.

Many electrophilic substitution reactions of 7-hydroxycoumarins, halogenation, nitration, formylation, azocoupling in the presence of weak base as well as Claisen and Fries rearragements yield preferably 8substituted 7-hydroxycoumarins [6-13]. 6-Substituted derivatives can be prepared by halogenation [14]. Claisen rearrangement [15], Elb's oxidation [16] and base-catalysed cyclization of acylmethyl ethers of 7hydroxycoumarins $[17,18]$ via the pyrone ring opening step.

Electrophilic substitution reactions of psoralens, e. g. Vilsmeier formylation and chloromethylation result in 4'-substitutied psoralens [19].

We have studied the azocoupling reaction of 7-hydroxycoumarins and psoralens based on the pyrone ring opening step. Psoralens have not been studied yet in this reaction. Azopsoralens seem to be a new series of biologically active photosensitive compounds.

\section{Results and discussion}

We carried out the azocoupling reaction of 7-hydroxy-4-methylcoumarin 1 with several parasubstituted aryldiazonium fluoroborates in $5 \%$ aqueous sodium hydroxide solution (Scheme I). The reaction 
yields only 6-arylazo-7- hydroxy-4-methylcoumarins $\underline{\text { 4a-e. }}$ Pyrone ring opening step generates phenoxide anion (Structure $\underline{2}$ ) where position 6 is strongly activated. Yields of the reaction depend on substituent in the para-position of aryldiazonium tetrafluoroborate (Table 1 ).

Scheme 1

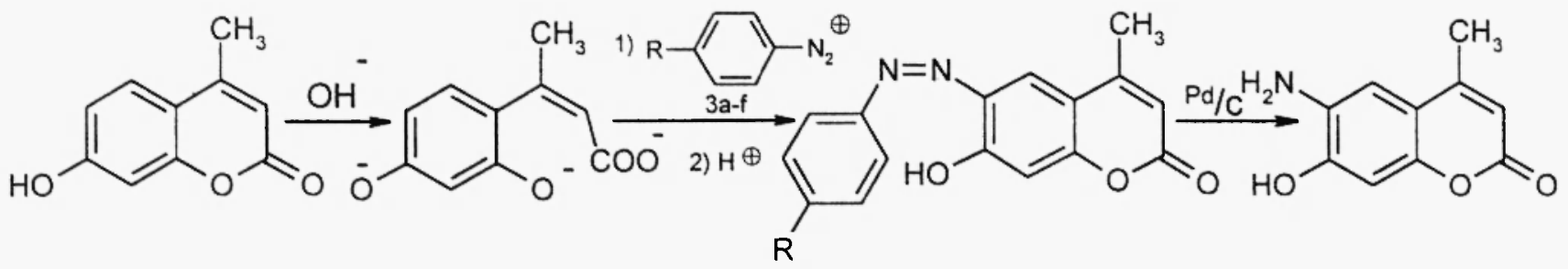

$\underline{1}$ $\underline{2}$

$\underline{\text { 4a-f }}$

Table 1. 6-Arylazocoumarins $\underline{4 a-f}$

\begin{tabular}{|c|c|c|c|c|c|c|}
\hline $\mathrm{R}$ & $\mathrm{H}$ & $\mathrm{CH}_{3}$ & $\mathrm{OCH}_{3}$ & $\mathrm{Cl}$ & $\mathrm{Br}$ & $\mathrm{NO}_{2}$ \\
\hline compound & $4 \mathrm{a}$ & $4 \mathrm{~b}$ & $4 \mathrm{c}$ & $4 \mathrm{~d}$ & $4 \mathrm{e}$ & $4 \mathrm{f}$ \\
\hline Yield, \% & 80 & 80 & 73 & 28 & 25 & 0 \\
\hline
\end{tabular}

Single peaks at 13.1-13.5 ppm are seen in spectra $\mathrm{I}_{\mathrm{H}}$ NMR of phenylazocoumarins $\underline{\text { 4a-e }}$ in accordance to their o-hydroxy-azo tautomeric forms.

We have studied effect of pyrone ring opening on azocoupling reaction of psoralens as well. Using psoralen $\underline{\mathbf{6}}$ and standard azocoupling reaction procedure, we have synthesized series of 5 '-arylazopsoralens 8a-e with good yields (Table 2). Position 5' seems to be much stronger activated than position 8 under ring opening (Scheme 2).

In accordance to this conclusion, our attempts to carry out azocoupling of psoralens in slightly-acidic or neutral conditions failed and starting psoralen was only recovered.

\section{Scheme 2}<smiles>[R]c1ccc(N=Nc2oc3cc4oc(=O)cc(C)c4cc3c2C)cc1</smiles> 
Table 2. Arylazopsoralens $\underline{8 \text { a-d }}$

\begin{tabular}{|c|c|c|c|c|}
\hline $\mathrm{R}$ & $\mathrm{H}$ & $\mathrm{CH}_{3}$ & $\mathrm{OCH}_{3}$ & $\mathrm{Cl}$ \\
\hline Compound & $8 \mathrm{a}$ & $8 \mathrm{~b}$ & $8 \mathrm{c}$ & $8 \mathrm{~d}$ \\
\hline Yield. \% & 85 & 82 & 70 & 50 \\
\hline
\end{tabular}

We have also compared the effect of pyrone ring opening on reactivity of positions $4^{\prime}$ and $5^{\circ}$ of psoralen in the azocoupling reaction. Psoralen 9 has been prepared with $30 \%$ overall yield by [19]. The azocoupling reaction of psoralen $\mathbf{9}$ (Scheme 3 ) in basic conditions starts with pyrone ring opening to produce dianion 10 where position 5' seems to be stronger activated by the phenoxide oxygen group. Azocoupling and acidification steps lead to final product 11 with $75 \%$ yield.

\section{Scheme 3}<smiles>Cc1cc(=O)oc2c(C)c3occc3cc12</smiles>

$\underline{9}$<smiles>C/C(=C/C(=O)[O-])c1cc2ccoc2c(C)c1[O-]</smiles><smiles>CCO[N+]1([O-])CCCCC1</smiles><smiles>COc1ccc(N=Nc2ccc([NH3+])cc2)cc1</smiles>

$\underline{10}$<smiles>Cc1cc2cc3c(C)cc(=O)oc3c(C)c2o1</smiles>

$\underline{11}$

We have used ${ }^{1}$ H NMR spectral data to define location of arylazo group in the psoralen $\underline{11}$. As one can see, introduction of arylazogroup to the position 6 of 4-methyl-7-hydroxycoumarin shifts peak H-5 for 0.7-0.8 ppm down field (Table 3). According to ${ }^{1} \mathrm{H}$ NMR spectra of compounds $\underline{\mathbf{8}}$, introduction of arylazogroup to the position 5 ' of 4, 4'-dimethylpsoralen shifts peak H-5 for 0.08-0.17 ppm down field (Table 4).

Table 3. Chemical shifts of proton $H-5$ in arylazocoumarins $\underline{1}$ and $\underline{4 a-e}$

\begin{tabular}{|l|c|c|c|c|c|c|}
\hline Coumarin & $\mathrm{l}$ & $4 \mathrm{a}$ & $4 \mathrm{~b}$ & $4 \mathrm{c}$ & $4 \mathrm{~d}$ & $4 \mathrm{e}$ \\
\hline $\mathrm{R}$ & $\mathrm{H}$ & $\mathrm{H}$ & $\mathrm{CH}_{3}$ & $\mathrm{OCH}_{3}$ & $\mathrm{Cl}$ & $\mathrm{Br}$ \\
\hline $\mathrm{H}-5$ & 7.38 & 8.18 & 8.14 & 8.10 & 8.17 & 8.17 \\
\hline$\delta \underline{4 a-e}-\delta \underline{1}$ & 0 & 0.8 & 0.76 & 0.72 & 0.79 & 0.79 \\
\hline
\end{tabular}


Table 4. Chemical shift of proton $H-5$ for set of psoralens $\underline{6}$ and $\underline{8 a-d}$

\begin{tabular}{|l|c|c|c|c|c|}
\hline Psoralen & 6 & $8 \mathrm{a}$ & $8 \mathrm{~b}$ & $8 \mathrm{c}$ & $8 \mathrm{~d}$ \\
\hline $\mathrm{R}$ & $\mathrm{H}$ & $\mathrm{H}$ & $\mathrm{CH} 3$ & $\mathrm{OCH} 3$ & $\mathrm{Cl}$ \\
\hline $\mathrm{H}-5$ & 7.68 & 7.83 & 7.76 & 7.76 & 7.85 \\
\hline$\delta \underline{\mathbf{8 a}-\mathbf{d}-\delta \underline{6}}$ & - & 0.15 & 0.08 & 0.08 & 0.17 \\
\hline
\end{tabular}

Therefore, we can expect peak $\mathrm{H}-5$ in the region $7.75-7.84 \mathrm{ppm}$ both for $4^{\circ}$-arylazo and for $5^{\circ}$ arylazopsoralens. On other hand for $4^{\prime}$-arylazopsoralen, proton $\mathrm{H}-5^{\prime}$ should appears at $8.42-8.50 \mathrm{ppm}$. In $5^{\prime}$ arylazopsoralen 'H NMR spectrum we can expect peak H-4' at 6.98-7.06 ppm.

Initial psoralen $\underline{9}$ shows peaks of protons $\mathrm{H}-4{ }^{\prime}, \mathrm{H}-5$ and $\mathrm{H}-5^{\prime}$ at $6.26,7.67$ and 7.70 ppm respectively. In ${ }^{1} \mathrm{H}$ NMR spectrum of $\underline{11}$ we found two singlets at 7.33 and $7.77 \mathrm{ppm}$, which correspond to signals $\mathrm{H}-4^{*}$ and $\mathrm{H}-5$ peaks respectively. Peak at $7.33 \mathrm{ppm}$ cannot correspond to proton $\mathrm{H}-5$, because it appears in 4.8dimethylpsoralen $\underline{9}$ at $7.67 \mathrm{ppm}$ and introduction of electronowithdrawing arylazogroup would shift this signal much more down field.

To find synthetic advantages of azocoupling reactions of coumarin derivatives based on the ring opening we reduced azocoumarin $\underline{4 a}$ for the amino derivative. Different reductive agents such as zinc powder with $\mathrm{HCl}$, sodium hydrosulfite and hydrogen with $5 \% \mathrm{Pd}$ on activated carbon catalyst have been used.

Reduction by $\mathrm{Zn}-\mathrm{HCl}$ in ethanol takes $2-3$ hours at $40-45^{\circ} \mathrm{C}$. Neutralization of the reaction mixture with aqueous ammonia solution gives aminocoumarin $\underline{\mathbf{5}}$ with $45 \%$ yield.

Reduction of $\underline{\mathbf{4 a}}$ by sodium hydrosulfite in $20 \%$ aqueous ammonia turned to be unsuccessful: it goes slow and provides a poor yield of coumarin $\underline{\mathbf{5}}$.

In opposite, reduction by hydrogen on $5 \%$ palladium activated carbon in ethanol takes only 3 hours at $40-45^{\circ} \mathrm{C}$ and provides aminocoumarin $\underline{\mathbf{5}}$ with $85 \%$ yield. Earlier coumarin_ $\underline{\mathbf{5}}$ has been prepared with $14 \%$ overall yield by nitration of coumarin $\underline{1}$ followed by separation of 6 - nitro (28\%) and 8-nitro (32\%) coumarins and reduction of 6-nitro derivative [17].

\section{Experimental}

'H NMR spectra were scanned with $200 \mathrm{MHz}$ Varian Gemini-2000 instrument. Chemical shifts are given in ppm. splitting constants $J$ are given in $\mathrm{Hz}$. Mass spectra were scanned on a SSQ- 710 ( Finnigan MAT) spectrometer at the energy of ionizing electrons equals to $70 \mathrm{eV}$. Thin layer chromatography was done on Silufol UV- 254 sheets with chloroform or chloroform- ethylacetate mixture as eluent. All melting points are uncorrected. 


\section{Azocoupling of hydroxycoumarins (general procedure).}

A mixture of $\underline{1}(10 \mathrm{mmol})$ and aryldiazonium tetrafluoroborate $(12 \mathrm{mmol})$ with $25 \mathrm{ml} 5 \% \mathrm{NaOH}$ solution was stirred at $0-5{ }^{\circ} \mathrm{C}$ for $2-4$ hours. Then the reaction mixture was acidified with hydrochloric acid to $\mathrm{pH}=5$. Formed precipitate was filtered off and purified by recrystallization from ethanol or by column chromatography (eluent-chloroform, silicagel 100-250 mesh). Yields, melting points and 'H NMR data of prepared coumarins $\underline{\mathbf{4 a}-\mathbf{e}}$ are listed below.

4a, 7-hydroxy-4-methyl-6-(phenylazo)coumarin, yield $80 \%, \mathrm{mp} 216-218^{\circ} \mathrm{C}$;

'H NMR: $\delta 2.49\left(\mathrm{~d}, 3 \mathrm{H}, \mathrm{J}_{\mathrm{CH} 3 . \mathrm{H} 3}=1.2,4-\mathrm{CH}_{3}\right) ; 6.20\left(\mathrm{q}, 1 \mathrm{H}, \mathrm{J}_{\mathrm{H} 3 . \mathrm{CH} 3}=1.2 \mathrm{~Hz}, \mathrm{H}-3\right) ; 6.91(\mathrm{~s}, 1 \mathrm{H}, \mathrm{H}-8): 7.50-7.60(\mathrm{~m}$, $\left.3 \mathrm{H}, 2 \mathrm{H}-3^{\prime}+\mathrm{H}^{\prime} 4^{\prime}\right) ; 7.90-8.00\left(\mathrm{~m}, 2 \mathrm{H}, \mathrm{H}-2^{\prime}\right) ; 8.18(\mathrm{~s}, 1 \mathrm{H}, \mathrm{H}-5): 13.35(\mathrm{~s}, 1 \mathrm{H}, \mathrm{OH})$.

MS: m/z (\%) 280(M $\left.\mathrm{M}^{+}, 100\right) ; 203\left(-\mathrm{C}_{6} \mathrm{H}_{5}, 35\right) ; 175\left(-\mathrm{C}_{6} \mathrm{H}_{5} \mathrm{~N}_{2}, 55\right) ; 147\left(-\mathrm{C}_{6} \mathrm{H}_{5} \mathrm{~N}_{2},-\mathrm{CO}, 20\right), 119\left(-\mathrm{C}_{6} \mathrm{H}_{5} \mathrm{~N}_{2}\right.$. $-2 \mathrm{CO}, 45) ; 91\left(-\mathrm{C}_{6} \mathrm{H}_{5} \mathrm{~N}_{2 .}-3 \mathrm{CO}, 20\right)$.

4b. 7-hydroxy-4-methyl-6-(p-methylphenylazo)coumarin, yield $80 \%$, mp $175-177^{\circ} \mathrm{C}$;

'H NMR: $\delta 2.45\left(\mathrm{~s}, 3 \mathrm{H}, \mathrm{CH}_{3}\right) ; 2.49\left(\mathrm{~d}, 3 \mathrm{H}, \mathrm{J}_{\mathrm{CH} 3,3}=1.0 \mathrm{~Hz}, 4-\mathrm{CH}_{3}\right) ; 6.18\left(\mathrm{q}, 1 \mathrm{H}, \mathrm{J}_{3, \mathrm{CH} 3}=1.0 \mathrm{~Hz}, \mathrm{H}-3\right) ; 6.89(\mathrm{~s}, 1 \mathrm{H}$, $\mathrm{H}-8$ ); 7.35(d, 2H, J $\left.3^{\prime} .2^{\prime}=8.8 \mathrm{~Hz}, \mathrm{H}-3^{\prime}\right) ; 7.77\left(\mathrm{~d}, 2 \mathrm{H}, \mathrm{j}_{2}\right.$ '. 3'=8.8 Hz, H-2'); 8.14(s, 1H, H-5); $13.42(\mathrm{~s}, 1 \mathrm{H}, \mathrm{OH})$.

MS: $\mathrm{m} / \mathrm{z}(\%) 294\left(\mathrm{M}^{+}, 78\right) ; 203\left(-\mathrm{CH}_{3} \mathrm{C}_{6} \mathrm{H}_{4}, 18\right) ; 175\left(-\mathrm{CH}_{3} \mathrm{C}_{6} \mathrm{H}_{4} \mathrm{~N}_{2}, 33\right) ; 147\left(-\mathrm{CH}_{3} \mathrm{C}_{6} \mathrm{H}_{4} \mathrm{~N}_{2},-\mathrm{CO}, 18\right)$, $119\left(-\mathrm{C}_{6} \mathrm{H}_{4} \mathrm{~N}_{2},-2 \mathrm{CO}, 35\right) ; 91\left(-\mathrm{C}_{6} \mathrm{H}_{4} \mathrm{~N}_{2},-3 \mathrm{CO}, 100\right)$.

4c, 7-hydroxy-6-(p-methoxyphenylazo)-4-methylcoumarin, yield $73 \%$, mp 223-2250 C;

${ }^{\prime} \mathrm{H}$ NMR: $\delta 2.49\left(\mathrm{~d}, 3 \mathrm{H}, \mathrm{J}_{\mathrm{CH} 3,3}=0.8 \mathrm{~Hz}, 4-\mathrm{CH}_{3}\right) ; 3.91\left(\mathrm{~s}, 3 \mathrm{H}, \mathrm{OCH}_{3}\right) ; 6.17\left(\mathrm{q}, 1 \mathrm{H}, \mathrm{J}_{3}, \mathrm{CH}_{3}=0.8 \mathrm{~Hz}, \mathrm{H}-3\right) ; 6.88(\mathrm{~s}$, $1 \mathrm{H}, \mathrm{H}-8) ; 7.03\left(\mathrm{~d}, 2 \mathrm{H}, \mathrm{J}_{3^{\prime}, 2^{\prime}}=8.8 \mathrm{~Hz}, \mathrm{H}-3^{\prime}\right) ; 7.85\left(\mathrm{~d}, 2 \mathrm{H}, \mathrm{J}_{2}^{\prime}, 3^{\prime}=8.8 \mathrm{~Hz}, \mathrm{H}-2\right.$ '); $8.10(\mathrm{~s}, 1 \mathrm{H}, \mathrm{H}-5) ; 13.39(\mathrm{~s}, 1 \mathrm{H}$, $\mathrm{OH})$.

MS: $\mathrm{m} / \mathrm{z}(\%) 310\left(\mathrm{M}^{+}, 100\right) ; 203\left(-\mathrm{CH}_{3} \mathrm{OC}_{6} \mathrm{H}_{4}, 7\right) ; 175\left(-\mathrm{CH}_{3} \mathrm{OC}_{6} \mathrm{H}_{4} \mathrm{~N}_{2}, 20\right) ; 147\left(-\mathrm{CH}_{3} \mathrm{OC}_{6} \mathrm{H}_{4} \mathrm{~N}_{2},-\mathrm{CO}\right.$, 7), $119\left(-\mathrm{CH}_{3} \mathrm{OC}_{6} \mathrm{H}_{4} \mathrm{~N}_{2}-2 \mathrm{CO}, 20\right) ; 91\left(-\mathrm{CH}_{3} \mathrm{OC}_{6} \mathrm{H}_{4} \mathrm{~N}_{2},-3 \mathrm{CO}, 15\right)$.

4d, 6-(p-chlorophenylazo)-7-hydroxy-4-methylcoumarin, yield $28 \%$, mp $240-242^{\circ} \mathrm{C}$;

'H NMR: $\delta 2.50\left(\mathrm{~d}, 3 \mathrm{H}, \mathrm{J}_{\mathrm{CH} 3,3}=0.8 \mathrm{~Hz}, 4-\mathrm{CH}_{3}\right) ; 6.21\left(\mathrm{q}, 1 \mathrm{H}, \mathrm{J}_{3, \mathrm{CH} 3}=0.8 \mathrm{~Hz}, \mathrm{H}-3\right) ; 6.92(\mathrm{~s}, 1 \mathrm{H}, \mathrm{H}-8) ; 7.52(\mathrm{~d}, 2 \mathrm{H}$, $\left.\mathrm{J}_{3^{\prime}, 2^{\prime}}=8.8 \mathrm{~Hz}, \mathrm{H}-3^{\prime}\right) ; 7.84\left(\mathrm{~d}, 2 \mathrm{H}, \mathrm{J}_{2}{ }^{\prime}, 3^{\prime}=8.8 \mathrm{~Hz}, \mathrm{H}-2^{\prime}\right) ; 8.17(\mathrm{~s}, 1 \mathrm{H}, \mathrm{H}-5) ; 13.12(\mathrm{~s}, 1 \mathrm{H}, \mathrm{OH})$.

MS: $\mathrm{m} / \mathrm{z}(\%) 316\left(\mathrm{M}^{+}{ }^{37} \mathrm{Cl}, 35\right) ; 314\left(\mathrm{M}^{+}{ }^{35} \mathrm{Cl}, 100\right) ; 203\left(-\mathrm{ClC}_{6} \mathrm{H}_{4}, 37\right) ; 175\left(-\mathrm{ClC}_{6} \mathrm{H}_{4} \mathrm{~N}_{2} 60\right) ; 147(-$ $\left.\mathrm{ClC}_{6} \mathrm{H}_{4} \mathrm{~N}_{2},-\mathrm{CO}, 26\right), 119\left(-\mathrm{ClC}_{6} \mathrm{H}_{4} \mathrm{~N}_{2}-2 \mathrm{CO}, 55\right) ; 91\left(-\mathrm{ClC}_{6} \mathrm{H}_{4} \mathrm{~N}_{2},-3 \mathrm{CO}, 32\right)$.

4e, 6-(p-bromophenylazo)-7-hydroxy-4-methylcoumarin, yield $25 \%$, mp 222-2240 C;

${ }^{1} \mathrm{H}$ NMR: $\delta 2.49\left(\mathrm{~d}, 3 \mathrm{H}, \mathrm{J}_{\mathrm{CH} 3,3}=1.0 \mathrm{~Hz}, 4-\mathrm{CH}_{3}\right) ; 6.2 \mathrm{l}\left(\mathrm{q}, 1 \mathrm{H}, \mathrm{J}_{3} . \mathrm{CH}_{3}=0.8 \mathrm{~Hz}, \mathrm{H}-3\right) ; 6.9 \mathrm{l}(\mathrm{s}, 1 \mathrm{H} . \mathrm{H}-8) ; 7.64-$ 7.80(m, 4H, H-Ar); 8.17(s, 1H, H-5) ); 13.12(s, 1H, OH). 
MS: $\mathrm{m} / \mathrm{z}(\%) 360\left(\mathrm{M}^{+}{ }^{81} \mathrm{Br}, 100\right) ; 358\left(\mathrm{M}^{+}{ }^{79} \mathrm{Br}, 92\right) ; 203\left(-\mathrm{BrC}_{6} \mathrm{H}_{4}, 48\right) ; 175\left(-\mathrm{BrC}_{6} \mathrm{H}_{4} \mathrm{~N}_{2} .91\right) ; 147-$ $\left.\mathrm{BrC}_{6} \mathrm{H}_{4} \mathrm{~N}_{2},-\mathrm{CO}, 33\right), 119\left(-\mathrm{BrC}_{6} \mathrm{H}_{4} \mathrm{~N}_{2 .}-2 \mathrm{CO}, 68\right) ; 91\left(-\mathrm{BrC}_{6} \mathrm{H}_{4} \mathrm{~N}_{2},-3 \mathrm{CO}, 45\right)$.

\section{Azocoupling of psoralens (general procedure).}

$10 \mathrm{mmol}$ of psoralen $\underline{6}$ were dissolved in minimal amount of hot ethanol. The solution was mixed with $20 \mathrm{ml} 5 \%$ aqueous $\mathrm{NaOH}$ solution (avoid ppt formation), cooled to $0-5^{\circ} \mathrm{C}$. Then $15 \mathrm{mmol}$ of aryldiazonium tetrafluoroborate was added to the prepared solution. Resulted reaction mixture was stirred at $0-5^{\circ} \mathrm{C}$ for $2-4$ hours with TLC test (eluent-chloroform) and was then acidified with diluted aqueous $\mathrm{HCl}$ solution. Formed precipitate was filtered and purified by recrystallization from ethanol or by column chromatography (eluent-chloroform, silicagel 100/250 mesh). Yields, melting points and 'H NMR data of prepared psoralens $\underline{\mathbf{8} \mathbf{a}-\mathbf{d}}$ and $\underline{\mathbf{1 1}}$ are listed below.

8a $, 4,4^{\prime}$-dimethyl-5'-phenylazopsoralen, yield $85 \%$, mp $180-182^{\circ} \mathrm{C}$;

${ }^{1} \mathrm{H}$ NMR $\left(\mathrm{CDCl}_{3}, \mathrm{~J} / \mathrm{Hz}\right): \delta 2.53\left(\mathrm{~s}, 3 \mathrm{H}, 4-\mathrm{CH}_{3}\right) ; 2.73\left(\mathrm{~s}, 3 \mathrm{H}, 4^{\prime}-\mathrm{CH}_{3}\right) ; 6.28(\mathrm{~s}, 1 \mathrm{H}, \mathrm{H}-3) ; 7.47(\mathrm{~s}, 1 \mathrm{H}, \mathrm{H}-8)$; 7.5(m, Hpara); 7.5(m, Hmeta); 7.83(s, 1H, 5-CH3); 7.98(m, Hortho).

MS: $\mathrm{m} / \mathrm{z}(\%) 318\left(\mathrm{M}^{+}, 100\right)$.

$\underline{\text { 8b }}$, 4, 4'-dimethyl-5'-(p-methylphenylazo)psoralen, yield $82 \%$, mp 228-230 ${ }^{\circ} \mathrm{C}$;

${ }^{\prime} \mathrm{H} \mathrm{NMR}\left(\mathrm{CDCl}_{3}, \mathrm{~J} / \mathrm{Hz}\right): 2.42\left(\mathrm{~s}, 3 \mathrm{H}, 4\right.$ ' $\left.-\mathrm{CH}_{3}\right) ; 2.49\left(\mathrm{~d}, 3 \mathrm{H}, \mathrm{J}_{\mathrm{CH} 3,3}=1.0,4-\mathrm{CH}_{3}\right) ; 2.67\left(\mathrm{~s}, 3 \mathrm{H}, \mathrm{p}-\mathrm{CH}_{3}\right) ; 6.23(\mathrm{q}, 1 \mathrm{H}$, $\left.\mathrm{J}_{3, \mathrm{CH} 3}=1.0, \mathrm{H}-3\right) ; 7.28\left(\mathrm{~d}, 2 \mathrm{H}, \mathrm{J}_{\mathrm{m}, 0}=8.8\right.$, Hortho); 7.45(s, $\left.1 \mathrm{H}, \mathrm{H}-8\right) ; 7.761(\mathrm{~s}, 1 \mathrm{H}, \mathrm{H}-5) ; 7.85\left(\mathrm{~d}, 2 \mathrm{H}, \mathrm{J}_{\mathrm{om}}=8.8\right.$, Hmeta).

MS: $\mathrm{m} / \mathrm{z}(\%) 332\left(\mathrm{M}^{+}, 100\right)$.

8c, 4, 4'-dimethyl-5'-(p-methoxyphenylazo)psoralen, yield $70 \%$, mp 206-207 ${ }^{\circ} \mathrm{C}$;

${ }^{1} \mathrm{H}$ NMR $\left(\mathrm{CDCl}_{3}, \mathrm{~J} / \mathrm{Hz}\right): 2.50\left(\mathrm{~d}, 3 \mathrm{H}, \mathrm{J}_{\mathrm{CH} 3,3}=1.0,4-\mathrm{CH}_{3}\right) ; 2.66\left(\mathrm{~s}, 3 \mathrm{H}, 4\right.$ '- $\left.\mathrm{CH}_{3}\right) ; 3.89\left(\mathrm{~s}, 3 \mathrm{H}, \mathrm{OCH}_{3}\right) ; 6.24(\mathrm{q}, 1 \mathrm{H}$, $\left.\mathrm{J}_{3, \mathrm{CH} 3}=1.0, \mathrm{H}-3\right) ; 6.99\left(\mathrm{~d}, 2 \mathrm{H}, \mathrm{J}_{\mathrm{m}, \mathrm{o}}=9.0\right.$, Hmeta); 7.46(s, $\left.1 \mathrm{H}, \mathrm{H}-8\right) ; 7.758(\mathrm{~s}, 1 \mathrm{H}, \mathrm{H}-5) ; 7.95\left(\mathrm{~d}, 2 \mathrm{H}, \mathrm{J}_{\mathrm{o}, \mathrm{m}}=9.0\right.$, Hortho).

MS: $\mathrm{m} / \mathrm{z}(\%) 348\left(\mathrm{M}^{+}, 100\right)$.

8d, $5^{\prime}$-(p-chlorophenylazo)-4,4'-dimethylpsoralen, yield $50 \%$, mp 167-168 ${ }^{\circ} \mathrm{C}$;

${ }^{1} \mathrm{H} \mathrm{NMR}\left(\mathrm{CDCl}_{3}, \mathrm{~J} / \mathrm{Hz}\right): 2.54\left(\mathrm{~d}, 3 \mathrm{H}, \mathrm{J}_{\mathrm{CH} 3,3}=1.0,4-\mathrm{CH}_{3}\right) ; 2.73\left(\mathrm{~s}, 3 \mathrm{H}, 4\right.$ '- $\left.\mathrm{CH}_{3}\right) ; 6.30\left(\mathrm{q}, 1 \mathrm{H}, \mathrm{J}_{3, \mathrm{CH} 3}=1.0\right) ; 7.48(\mathrm{~d}$, $2 \mathrm{H}, \mathrm{J}_{\mathrm{m} . \mathrm{o}}=8.8$, Hmeta); 7.50(s, $\left.1 \mathrm{H}, \mathrm{H}-8\right) ; 7.85(\mathrm{~s}, 1 \mathrm{H}, \mathrm{H}-5) ; 7.93\left(\mathrm{~d}, 2 \mathrm{H}, \mathrm{J}_{\mathrm{o} . \mathrm{m}}=8.8\right.$, Hortho).

MS: $\mathrm{m} / \mathrm{z}(\%) 352\left(\mathrm{M}^{+}, 100\right)$. 
MS: $\mathrm{m} / \mathrm{z}(\%) 352\left(\mathrm{M}^{+}, 100\right)$.

11, 4, 8-dimetliyl-5 -(p-methoxyphenylazo)psoralen, yield $75 \%$, mp 192-193 ${ }^{\circ} \mathrm{C}$;

${ }^{\mathrm{H}} \mathrm{H} \mathrm{NMR}\left(\mathrm{CDCl}_{3}, \mathrm{~J} / \mathrm{Hz}\right): 2.52\left(\mathrm{~d}, 3 \mathrm{H}, \mathrm{J}_{\mathrm{CH} 3.3}=1.0,4-\mathrm{CH}_{3}\right) ; 2.69\left(\mathrm{~s}, 3 \mathrm{H}, 8-\mathrm{CH}_{3}\right) ; 3.92\left(\mathrm{~s}, 3 \mathrm{H}, \mathrm{OCH}_{3}\right): 6.29(\mathrm{q} .1 \mathrm{H}$. $\left.\mathrm{J}_{3 . \mathrm{CH} 3}=1.0, \mathrm{H}-3\right) ; 7.03\left(\mathrm{~d}, 2 \mathrm{H}, \mathrm{J}_{\mathrm{m}, 0}=8.0\right.$, Hmeta); 7.34(s, $\left.1 \mathrm{H}, \mathrm{H}-4^{\circ}\right) ; 7.77(\mathrm{~s}, 1 \mathrm{H}, \mathrm{H}-5) ; 7.98\left(\mathrm{~d}, 2 \mathrm{H}, \mathrm{J}_{\mathrm{om}}=8.0\right.$, Hortho);

MS: $\mathrm{m} / \mathrm{z}(\%) 348\left(\mathrm{M}^{+}, 100\right)$.

\section{Reduction of arylazocoumarins $\underline{4 a}$.}

A mixture of $400 \mathrm{mg} \underline{4 a}$ and $50 \mathrm{mg}$ of $5 \% \mathrm{Pd} / \mathrm{C}$ in minimal amount of ethanol was heated to $45^{\circ} \mathrm{C}$. Hydrogen gas has been passed through the stirred reaction mixture for 3 hours until the solution becomes light green. Then the reaction mixture was filtered and ethanol was evaporated from filtrate. Remained solid mass was recrystallized from ethanol to produce light green crystals. 6-amino-7-hydroxy-4-methylcoumarin $\underline{\mathbf{5}}$, yield $85 \%$, mp $251-253^{\circ} \mathrm{C}$;

${ }^{1} \mathrm{H}$ NMR: 2.26(s, 3H, $\left.\mathrm{CH}_{3}\right)$; 5.95(s, 1H, H-3); 6.56(s, 1H, H-8); 5.90(s, $\left.1 \mathrm{H}, \mathrm{H}-5\right)$.

MS: $\mathrm{m} / \mathrm{z}(\%) 191\left(\mathrm{M}^{+}, 100\right)$.

\section{Conclusion}

The pyrone ring opening step has been used for regioselective synthesis of 6-arylazo-7hydroxycoumarins $\underline{4}$ and 5 -arylazopsoralens $\underline{8}$ and $\underline{11}$.

\section{Acknowledgements}

This work was generously funds by the Highest Education State Committee of Russian Federation (program "Fine Organic Synthesis") and by the Otto Bremer Foundation (via Prof. Ed. Carberry project).

\section{Reference}

(1) R. A. Finnegan, K. E. Merkel and N. Black, J. Pharm. Sci. 61, 1599 (1972).

(2) L. W. Wattenburg, L. K. T. Lam and A. V. Fladmore, Cancer Res. 39, 1651 (1979).

(3) R.B. Silverman, J.Am. Chem. Soc. 103, 3910 (1981).

(4) A. Maxwell, Molecular Microbiology 9, 681 (1993).

(5) J. A. Parrish, R. S. Stern, M. A Pathak and T. B. Fitzpatrick, in "The Science of Photomedicine", J. D. Regan and J. A. Parrish Eds., Plenum Publishing Co., New York, 595 (1984). 
(7) N. M. Shah and D. H. Mehta, J. Ind. Chem. Soc. $\underline{3}$ 1. 784 (1954).

(8) A. N. Nesmeyanov, A. F. Vompe, T. S. Zaverich, D. D. Smolin, J. Gen. Chem. USSR (Engl. Transl) 7. 2767 (1937).

(9) Dean R. Bender. David Kanne, Ianice D. Frazier and Henry Rapoport, J. Org. Chem. 48. 2709-2719 (1983).

(10) T. S. Gore and P. K. Inamdar, Indian J. Chem. 29, 499 (1973).

(11) B. Krishnaswamy, and T. R. Seshadri, Proc. Indian Acad. Sci.(A), 쓰, 43 (1941).

(12) V. F. Traven, D. V. Kravtchenko, T. A. Chibisova, S. V. Shorshnev, R. Eliason and D. H. Wakefild, Heterocycl.Communications. 2, 345 (1996).

(13) V. K. Ahuja, K. L. Kapor and N. K. Ray, Indian J. Chem. 11, 458 (1972).

(14) E. Zubia, F. R. Luis, G. M. Massanet and I. G. Collado, Tetrahedron 48, 4239 (1992).

(15) N. Cairns, L. M. Harwood, D. P. Astles, and A. Orr, J.Chem.Soc., Chem.Commun. 182(1986).

(16) R. D. Desai and J. V. Parchi, J.Indian Chem. Soc. 33, 661 (1956).

(17) J. K. Macleod, B. R. Worth and R. J. Wells, Aust. J. Chem. 31, 1533 (1978).

(18) V. F. Traven, R. V. Rozhkov, A. Y. Tolmachev, N. A. Kuznezova, N. Y. Podhalusina and E. A. Carberry, Heterocycl. Communications $\underline{3}, 339$ (1997).

(19) K. D. Kaufman, D. J. Erb, T. M. Blok, R. W. Karlson, D. J. Knoechel, L. McBride, and T. Zeitlow, J. Heterocyclic Chem. 19, 1051 (1982).

(20) V. V. Somayajulu and N. V. Subba, F. A. Rao, Sci. Proc. Indian Acad. Sci $\underline{61}, 177$ (1965).

\section{Received on August 2, 1998}

